\title{
Trends in Supersonic Separator design development
}

\author{
Rami Ali Altam ${ }^{1}$, Tamiru Alemu Lemma ${ }^{1, *}$, and Shiferaw Regassa Jufar ${ }^{1}$ \\ ${ }^{1}$ Universiti Teknologi PETRONAS, 32610 Seri Iskandar, Perak Darul Ridzuan, Malaysia.
}

\begin{abstract}
Supersonic separator is a new technology with applications in hydrocarbon dew pointing and gas dehydration which can be used to condensate and separate water and heavy hydrocarbons from natural gas. Many researchers have studied the design, performance and efficiency, economic viability, and industrial applications of these separators. The purpose of this paper is to succinctly review recent progress in the design and application of supersonic separators and their limitations. This review has found that while several aspects of this study are well studied, considerable gaps within the published literature still exists in the areas such as turndown flexibility which is a critical requirement to cater for variation of mass flow and since almost all the available designs have a fixed geometry and therefore cannot be considered suitable for variable mass flow rate, which is a common situation in actual site. Hence, the focus needs to be more on designing a flexible geometry that can maintain a high separation efficiency regardless of inlet conditions and mass flow variations. This review is focusing only on the design and application of the supersonic separators without going through the experimental facilities, industrial platform, pilot plants as well as theoretical, analytical, and numerical modelling.
\end{abstract}

\section{Introduction}

The presence of $\mathrm{CO}_{2}$ in a natural gas mixture has a negative effect. As such, (i) it reduces heating value of the natural gas, (ii) it causes corrosion of the pipes and hence corrosionresistant materials have to be used which leads to a costly facility, (iii) high $\mathrm{CO}_{2}$ production requires separation process and huge storage facilities. However, the high capital cost for the Acid Gas Removal System (AGRS) considered being the major factor obstructing the monetization of a high $\mathrm{CO}_{2}$ gas field. Therefore, several technologies and processes have been used and evaluated by the industry which have the potential to remove $\mathrm{CO}_{2}$ from the natural gas. Each of these methods has its landscape of performance, energy requirement and system footprint [1], namely; amine and membrane which however were economically impossible. Besides, these technologies were technically non-viable as $\mathrm{CO}_{2}$ proportion gets higher [2]. Extensive studies then introduced a new technology called 'vortex tube'. This technology has been developed on the pressure difference principle. This principle is employed to a nozzle pressure that has hot and cold ends, these ends create a differential

$\overline{{ }^{1} \text { Corresponding author: tamiru.lemma@utp.edu.my }}$ 
pressure state, which is used in the supersonic flow. The role of supersonic flow is to create a low-temperature area inside the nozzle. This process can separate $\mathrm{CO}_{2}$, sulfur and other impurities from the natural gas that consist of heavier hydrocarbons. The credit for this goes to a physical principle called 'centrifugal force' which is caused by the swirling flow [3].

The supersonic separation unit consists of (i) Casing, (ii) swirling device, (iii) supersonic nozzle, (iv) liquid extraction and (v) diffuser. As shown in Fig. 1.

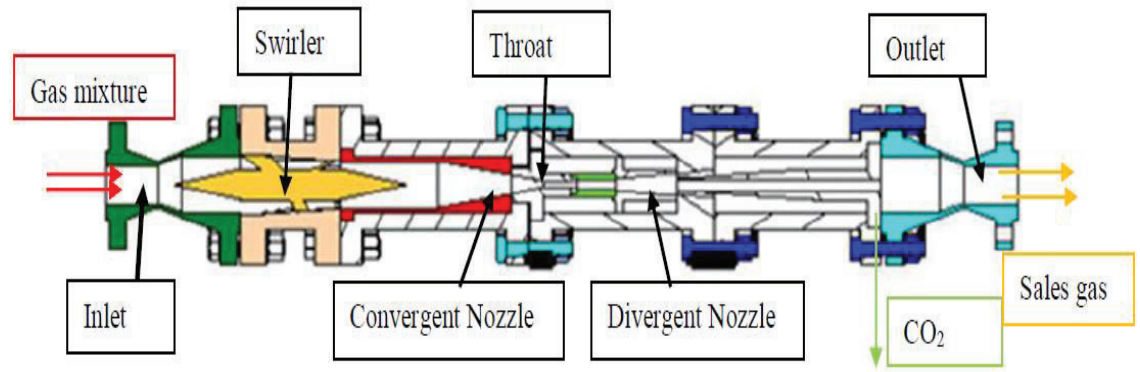

Fig. 1. Current supersonic separator UTP patent design.

One of the supersonic separation advantages is that it is more efficient in extracting dissolved impurities in natural gas. Moreover, supersonic separation unit has no rotating parts. Hence it needs less compression power which simplifies its scheme, footprint, and maintenance. Also, supersonic separation unit is cost-efficient due to its small and compact design, it also requires less operating cost.

There are several technologies by which $\mathrm{CO}_{2}$ can be separated. Two technologies are considered the most recent and efficient $\mathrm{CO}_{2}$ separation devices; namely, the $3 \mathrm{~S}$ supersonic separator and the second one is the Twister separator. A detailed review of the literature is presented in the sections that follow.

\section{Geometry of nozzle}

The Laval Nozzle is a tube of an hourglass shape that is pinched in the middle. It uses the principle of the Joule-Thompson effect where expanding the pressurized gas to low pressure at constant enthalpy results in transforming the potential energy (temperature and pressure) into kinetic energy (velocity) that eventually leads to accelerating the gas to a supersonic speed [4]. The Laval Nozzle is composed of three sections: expander, cyclone separator, and compressor as in Fig. 2.

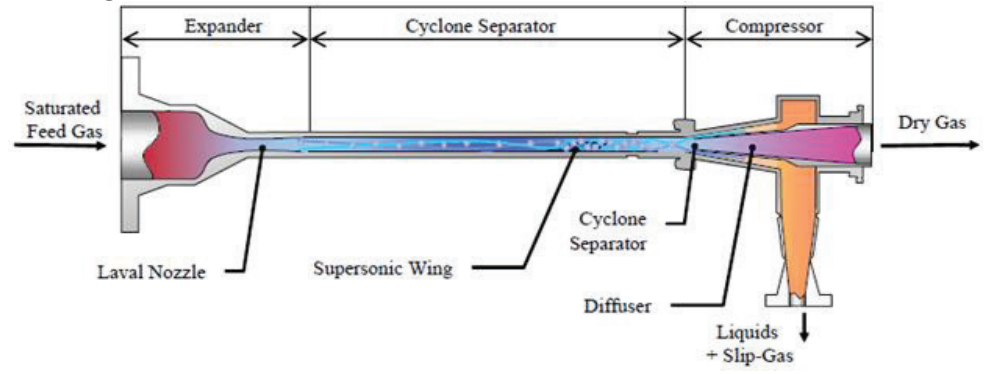

Fig. 2. Scheme of supersonic swirling separator(Twister-I) [4].

1. The expander (convergent section)

The expander (subsonic zone) is located at the inlet section. Where the swirling generator located in the expander is composed of several blades tangent to the nozzle. The gas flow 
passes through these blades leading the gas flow to accelerate and enter the nozzle with the tangential velocity of a particular value. The pressure and temperature are decreasing due to the supersonic speed of the wet gas in the expander. Where the drop of the temperature results in condensation of the water vapors existing in the gas $[5,6]$.

2. The Cyclone Separator (throat section)

After the expansion, in the cyclone separator (critical zone) where the already formed droplets are being separated to the walls due to the centrifugal force that can reach values up to $500000 \mathrm{~g}$ causing the cyclonic separation [5]. The droplets separated on the walls are then forming a thin film which is moving in the direction of the flow.

3. The Compressor (divergent section)

The Laval Nozzle ends with the compressor (supersonic zone). A separation channel is located in the compressor to separate the thin water film on the walls from the dry gas stream. Compressor part leads to the diffuser in order to recover the initial pressure of the gas where a shock wave is produced. The shock wave is generated by changing the velocity from supersonic to sonic speed. From here, the gas is slowed down in the diffuser that results in recovering about $(65-80 \%)$ of the initial pressure [7].

In the Laval nozzle, the inlet diameter must be greater than $\sqrt{5}$ of the throat diameter, in order to obtain the sonic speed at the throat [8]. On the other hand, the throat diameter should be equal to or smaller than the convergent length based on Wen et al. [5, 9-14] calculations where the Foelsch's analytical calculation [15] was used to compute the dimensions of the convergent section of the Laval nozzle in order to obtain the sound speed in the throat area and generate the stable supersonic flows. The recommended geometry is governed by:

$\left\{\begin{array}{lll}\frac{D-D_{c r}}{D_{1}-D_{c r}}=1-\frac{1}{X_{m}^{2}}\left(\frac{x}{L}\right)^{3} & \text { for } & \left(\frac{x}{L} \leq X_{m}\right) \\ \frac{D-D_{c r}}{D_{1}-D_{c r}}=\frac{1}{\left(1-X_{m}\right)^{2}}\left(1-\frac{x}{L}\right)^{3} & \text { for } & \left(\frac{x}{L}>X_{m}\right)\end{array}\right\}$

For converging-diverging nozzle, the optimum design proposed by Betting [16] dictates that $50 \leq \mathrm{L}_{2}\left(\mathrm{D}_{2}-\mathrm{D}_{\mathrm{cr}}\right) \leq 220$. Many of the designs use straighteners in the substantially gaseous region. However, the decision on the geometry and location of this component is still not clear.

\section{Twister BV}

Prast et al. [17] who was associated with Twister BV, studied the nucleation and growth of droplets in a 2D supersonic Laval nozzle. Twister separator which is based on air drying device was originally developed by Stork Product Engineering BV in 1989 [18]. Twister BV company is a joint venture between the Beacon Group and Shell launched in the year 2000 for developing the first generation of supersonic separators, trademarked Twister [19]. This model used a deflecting blade in the supersonic section of the nozzle to generate the swirl required for condensed particles separation and is now referred to as Twister-I as illustrated in Fig. 2. However, Twister design is reported to have a temperature drop as low as $\left(-40^{\circ} \mathrm{C}\right)$ with a corresponding pressure drop of up to $30 \%$. But then, a significant portion of the gas leaves with the mist, forcing the design to have a secondary separator called degassing vessel. Erosion of the wing or deltoid plate paneled to the inside wall downstream to the throat is a further drawback that is making the swirl action prone to extinction in a short period of time. The residence time for the droplets is also short rendering the separation performance very low. Therefore, the design of the nozzle was optimized by including a central body and an annular nozzle configuration [20]. The swirl generation was also moved upstream, in the subsonic part of the nozzle inlet (Twister-II) as showed in Fig. 3. 


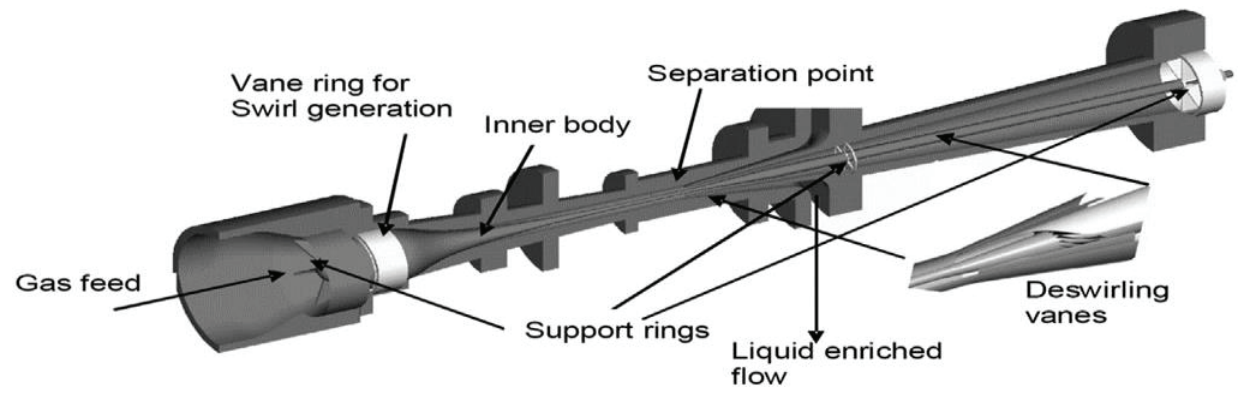

Fig. 3. Scheme of supersonic swirling separator (Twister-II) [21].

The improved design is being used by PETRONAS and Sarawak Shell Berhad (SSB). Nevertheless, inability to handle variable mass flow rates and high-pressure loss (30\%) are issues yet to be resolved.

\section{5 supersonic separator}

3S separator is supersonic separator designed by TransLang Technologies Limited which is an engineering services company. The $3 \mathrm{~S}$ supersonic separator is a hybrid of the two Twister devices. This device has a swirl generator in the subsonic entrance to the nozzle and has no central body as in Fig. 4.

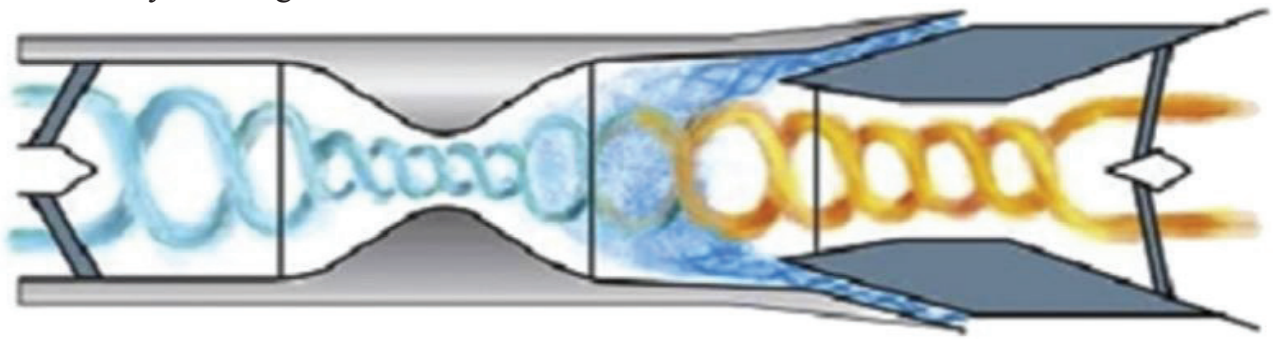

Fig. 4. 3-S separation [21].

3S Separator uses the fundamental principle of adiabatic expansion through a Laval nozzle. Additionally, however, a swirler is included to increase the centrifugal force in the separation zone. A de-swirler or directing vanes at the outlet is another feature that makes it different from other designs. As reported, a temperature drop of up to $\left(-70^{\circ} \mathrm{C}\right)$ is possible with a corresponding pressure ratio of about 2.5 and Mach numbers in the range of 1.5 to 2 . Because of the low temperature, the potential use of $3 \mathrm{~S}$ extends to LNG applications, $\mathrm{CO}_{2}$ extraction, and ethane recovery and the cryogenic temperature for natural gas is within the capacity limit of the separator. $3 \mathrm{~S}$ is patented in many countries including US, Canada, and Netherlands. Additionally, the $3 \mathrm{~S}$ separator was capable of dew pointing lean gases where Joule Thompson systems were ineffective. Some of these accomplishments were published in an article in the Oil and Gas Journal [22]. However, it seems at this point that there have been no further contributions from this group to the technology available from Twister.

\section{Garrett design [23]}

The separator is featured by supersonic expansion of a multi-component gas in a planar bend. The bend is shaped to have converging and diverging sections with an intervening minimum throat area. The condensed fluid is collected through a permeable wall as shown in Fig. 5. 


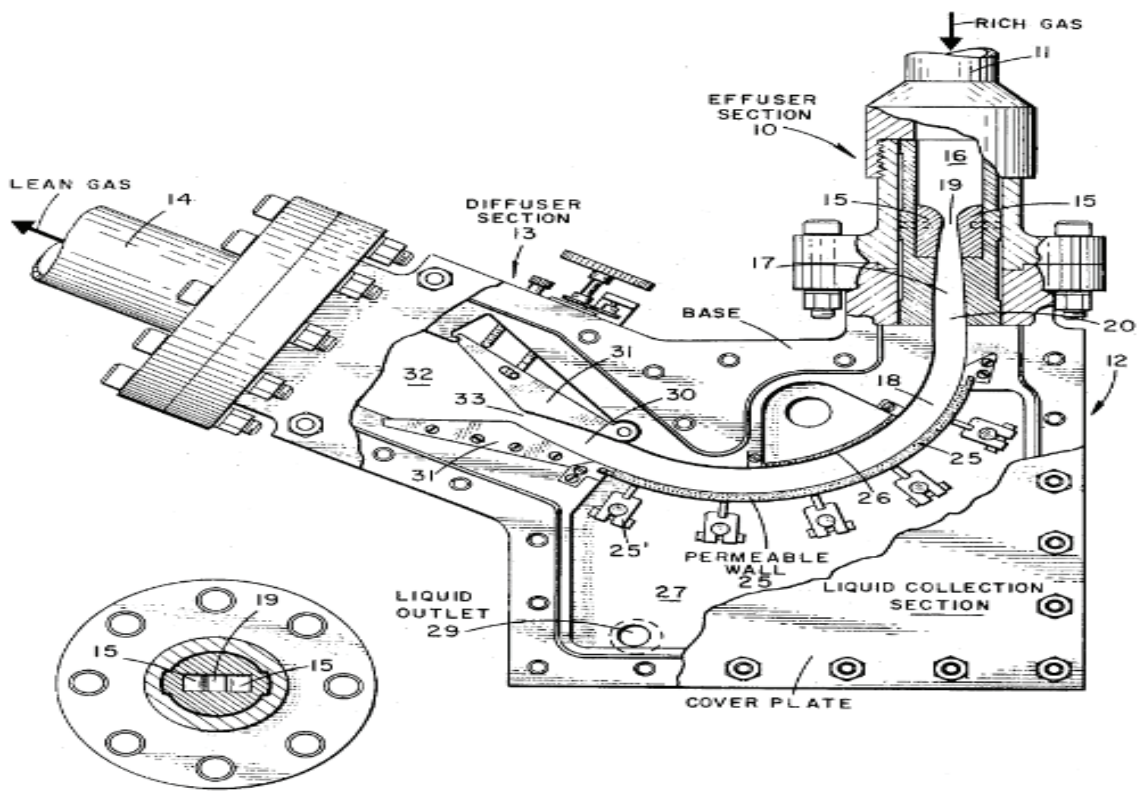

Fig. 5. Supersonic flow separator by Garrett [23].

The design is unique in the sense that it is equipped with (i) a mechanism to adjust size of the flow region and fully control the starting of supersonic flow and also the location of shockwave for maximum separation efficiency, and (ii) a permeable wall with electrostatic field to enhance particle ejection and droplet coalescence. One drawback of this design, however, is that the separation performance depends on the radius of curvature of the duct, which in turn is affected by the presence of unwanted shockwaves.

\section{Keisuke design [24]}

Designed to separate two or more gaseous mixtures, it has a converging-diverging nozzle with a swirler at the inlet, and two vacuum pumps connected to the exits. The oblique shock in the expansion zone is reported to have used for vaporizing the condensate back to the gaseous state. As documented by other authors, however, the design is featured by highpressure drop due to the long axial distance. It also needs sufficient energy to sustain strong swirl throughout the separation zone.

\section{Van Holten design [25]}

Unlike Garret Design, the separator by Val Holten has a cylindrical shape that converges to a nozzle and diverges into a swirl nozzle. The gas that enters to the separator at subsonic speed first flows through a converging section and then expands to supersonic speed in the diverging section of the separator. Plate-like elements attached to the inside wall and protruding radially into the flow imparts a swirl to the supersonic flow. The lean gas at supersonic speed is discharged through a centrally arranged pipe. Condensate reach mixture, on the other hand, leaves the separation zone through a diverging section where a suction fan is installed to draw out the fluid. This design suffers from erosion of the deltoid plates and the energy it needs to run the fan. Furthermore, there is no mechanism to deal with variable mass flow rate. According to the discussion in Betting et al. [16], Van Holten's separator has low efficiency due to the presence of shock waves in the first and second outlets. Which in 
other words is that substantial amount of the condensed gas leaves through the secondary outlet.

\section{Borissov design [26]}

Two version of Borissov designs have been reported. The first design called SUSTOR-I entails a plug or central body and inlet manifold where the swirl is initiated using guide vanes, as described in Fig. 6 (a). Some of the features are: (i) temperature drop up to $\left(-73^{\circ} \mathrm{C}\right)$, (ii) tangential inlet and tangential exit, (iii) reduced pressure drop for an equivalent temperature reduction, (iv) liquid droplets attached to the sidewalls by centrifugal action are removed through slits, (v) dry gas that is spirally expanding in the separator leaves the vortex chamber through a tangential outlet. The second design called SUSTOR-II involves a chamber consists of two disc-like parts (marked by the A-A and B-B cross-sections in Fig. 6 (b)) and the converging-diverging nozzle located in between. SUSTOR-II advantage is that the tangential exit is thought to have mitigated the high-pressure loss in SUSTOR-I. Nevertheless, the cast geometry is relatively complex.

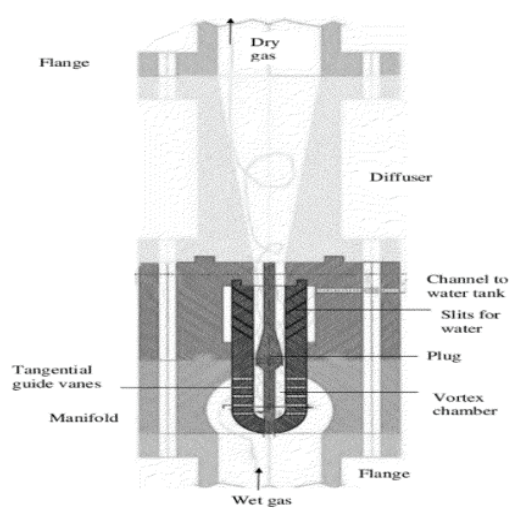

(a)

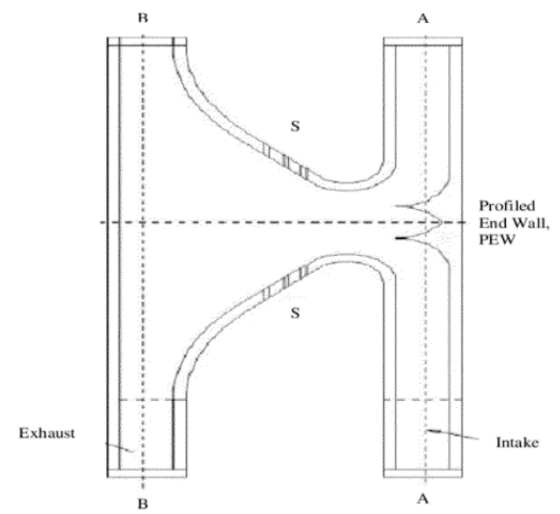

(b)

Fig. 6. Schematics of the (a) SUSTOR-I and (b) SUSTOR-II Separators [26].

\section{Wen et al. design [27]}

Wen and his colleagues have worked on the analysis and modelling of the supersonic separators over the last few years. This design has a cylindrical casing starting from inlet flange all the way to the expanding pipe. Akin to Twister-II and SUSTOR-I, it also has a central body and swirl generating blades. However, the shape of the central body is different, and the longitudinal cross-section has ellipsoid shape at the inlet while the rest of the edges are straight lines. The presence of a swirler at the inlet and resistive vortex vanes at the central outlet also making the design similar to $3 \mathrm{~S}$ separator. In terms of structure, is reported simple. However, the claim that it runs stably and has high efficiency is hard to verify for, and there is limited information on its performance and working range. Additionally, the design is fixed geometry and therefore cannot be considered suitable for variable mass flow rate, which is a common situation in actual site. 


\section{Beijing university of technology [28]}

A supersonic separator test apparatus with a throat diameter of $10 \mathrm{~mm}$ was built with stainless steel. The separator has no central body, in which a swirl generator was located downstream of the nozzle and in the supersonic flow region. The device also has a total of seven thermocouple probes along the nozzle, humidity and temperature sensors, pressure gauges, flow meters at the inlet and dry gas outlet of the separator, and also an extra pressure gauge at the wet outlet.
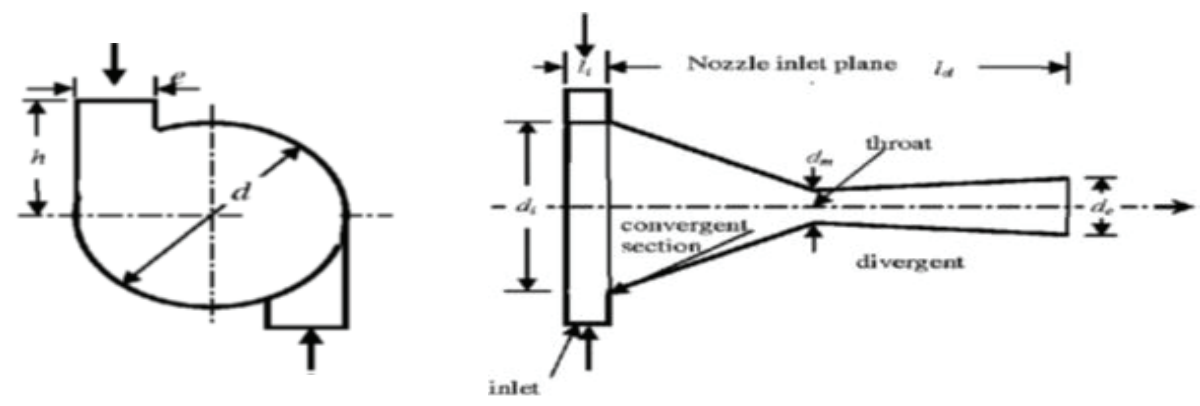

Fig. 7. Tangential inlet openings that generate the swirl [29].

As showed in Fig. 7, the flow is introduced as a tangential flow by passing through the two symmetrical inlet openings in which the swirl generator was relocated upstream to the subsonic section and also the blade system to generate swirl is not needed [29]. The dimensions of the two openings can be adjusted in order to increase or decrease the inlet velocity. However, changing the size of the inlet openings had no effect on the flow rate. Another factor to consider in this design was the strong gradients in tangential velocity near the axis of the nozzle.

\section{Discussion}

Table 1 shows the comparisons between the available designs of the supersonic separators. In terms of the main features, it is clear that almost all the separators contain a swirler generator installed except for Garrett design. On the other hand, Twister, SUSTOR-I and Wen et al. designs have a central body inside the Laval nozzle in order to increase the separation performance. Another main feature is the temperature drop which found to be the lowest of $\left(-70^{\circ} \mathrm{C}\right)$ in the $3 \mathrm{~S}$ separator and $\left(-73^{\circ} \mathrm{C}\right)$ in the SUSTOR separator. Garrett separator has a unique design with electrostatic field to enhance the separation and adjustable flow region that might be able to handle the flow rate variations. SUSTOR separator can also be scaled down for any rate of the gas flow. Regarding the limitation, almost all the designs suffer from the high-pressure losses and the turndown flexibility problem. SUSTOR-II considered being the latest and best design available recently. SUSTOR-II was able to minimize the pressure losses and solve the turndown flexibility. However, the cast geometry is relatively complex. 
Table 1. Comparison between the available supersonic separators designs.

\begin{tabular}{|c|c|c|}
\hline Design & Main features & Limitations \\
\hline TWISTER BV [7] & $\begin{array}{l}\text { - Deflecting blade to generate the } \\
\text { swirl. } \\
\text { - Central body in order to increase } \\
\text { the separation performance. } \\
\text { - A temperature drops as low as (- } \\
\left.40^{\circ} \mathrm{C}\right) \text {. }\end{array}$ & $\begin{array}{l}\text { Inability to handle variable mass } \\
\text { flow rates and high-pressure loss } \\
\text { up to } 30 \% \text {. }\end{array}$ \\
\hline $\begin{array}{l}\text { 3S Supersonic } \\
\text { Separator [22] }\end{array}$ & $\begin{array}{l}\text { - Hybrid of the two Twister } \\
\text { devices. } \\
\text { - A swirler is included. } \\
\text { - A de-swirler or directing vanes } \\
\text { at the outlet. } \\
\text { Temperature drop of up to (- } \\
\left.70^{\circ} \mathrm{C}\right) \text {. }\end{array}$ & $\begin{array}{l}\text { - Fixed geometry that lacks the } \\
\text { ability to handle the variable } \\
\text { mass flow rates. }\end{array}$ \\
\hline $\begin{array}{c}\text { Garrett Design } \\
{[23]}\end{array}$ & $\begin{array}{l}\text { - Featured with a planar bend } \\
\text { contains the converging and } \\
\text { diverging sections with an } \\
\text { intervening minimum throat } \\
\text { area. } \\
\text { A permeable wall to collect the } \\
\text { condensed fluid. } \\
\text { - Adjustable flow region. } \\
\text { - Electrostatic field to enhance the } \\
\text { separation. }\end{array}$ & $\begin{array}{l}\text { - The separation performance } \\
\text { depends on the radius of } \\
\text { curvature of the duct, which in } \\
\text { turn is affected by the presence } \\
\text { of unwanted shock waves. }\end{array}$ \\
\hline $\begin{array}{c}\text { Keisuke Design } \\
{[24]}\end{array}$ & $\begin{array}{l}\text { A converging-diverging nozzle } \\
\text { with a swirler at the inlet and two } \\
\text { vacuum pumps connected to the } \\
\text { exits. }\end{array}$ & $\begin{array}{l}\text { - The design is featured by high- } \\
\text { pressure drop due to the long } \\
\text { axial distance. } \\
\text { It needs sufficient energy to } \\
\text { sustain strong swirl throughout } \\
\text { the separation zone. }\end{array}$ \\
\hline $\begin{array}{c}\text { Van Holten Design } \\
{[25]}\end{array}$ & $\begin{array}{l}\text { - A cylindrical shape that } \\
\text { converges to a nozzle and } \\
\text { diverges into a swirl nozzle. } \\
\text { Plate-like elements attached to } \\
\text { the inside wall and protruding } \\
\text { radially into the flow imparts a } \\
\text { swirl to the supersonic flow. } \\
\text { The gas at supersonic speed is } \\
\text { discharged through a centrally } \\
\text { arranged pipe. } \\
\text { A suction fan is installed in the } \\
\text { diverging section to draw out the } \\
\text { fluid. }\end{array}$ & $\begin{array}{l}\text { - The design suffers from erosion } \\
\text { of the deltoid plates and the } \\
\text { energy it needs to run the fan. } \\
\text { There is no mechanism to deal } \\
\text { with variable mass flow rate. } \\
\text { Has low efficiency due to the } \\
\text { presence of shock waves in the } \\
\text { first and second outlets. }\end{array}$ \\
\hline $\begin{array}{l}\text { SUSTOR-I\&II } \\
{[26]}\end{array}$ & $\begin{array}{l}\text { - SUSTOR-I entails a plug or } \\
\text { central body and inlet manifold } \\
\text { where the swirl is initiated using } \\
\text { guide vanes. } \\
\text { - A temperature drops up to (- } \\
73^{\circ} \mathrm{C} \text { ). } \\
\text { Reduced pressure drop for an } \\
\text { equivalent temperature } \\
\text { reduction. }\end{array}$ & $\begin{array}{l}\text { - The cast geometry is relatively } \\
\text { complex. }\end{array}$ \\
\hline
\end{tabular}




\begin{tabular}{|c|c|c|}
\hline & $\begin{array}{l}\text { - The liquid is removed through } \\
\text { slits. } \\
\text { Dry gas leaves through a } \\
\text { tangential outlet. } \\
\text { The tangential exit in SUSTOR- } \\
\text { II is thought to have mitigated } \\
\text { the high-pressure loss in the } \\
\text { SUSTOR-I design. } \\
\text { - Can be scaled down for any rate } \\
\text { of the gas flow. }\end{array}$ & \\
\hline $\begin{array}{c}\text { Wen et al. Design } \\
{[27]}\end{array}$ & $\begin{array}{l}\text { - A cylindrical casing is starting } \\
\text { from inlet flange all the way to } \\
\text { the expanding pipe. } \\
\text { - A central body and swirl } \\
\text { generating blades. } \\
\text { - Simple structure. }\end{array}$ & $\begin{array}{l}\text { - The claim that it runs stably and } \\
\text { has high efficiency is hard to } \\
\text { verify for, and there is limited } \\
\text { information on its performance } \\
\text { and working range. } \\
\text { Fixed geometry which is unable } \\
\text { to handle variable mass flow } \\
\text { rates. }\end{array}$ \\
\hline $\begin{array}{l}\text { Design by Beijing } \\
\text { University of } \\
\text { Technology [28] }\end{array}$ & $\begin{array}{l}\text { - No central body. } \\
\text { - Swirl generator located } \\
\text { downstream of the nozzle. } \\
\text { Contains total of seven } \\
\text { thermocouple probes along the } \\
\text { nozzle. } \\
\text { Tangential flow by passing } \\
\text { through two symmetrical inlet } \\
\text { openings that can be adjusted to } \\
\text { increase or decrease the inlet } \\
\text { velocity. }\end{array}$ & $\begin{array}{l}\text { - Changing the size of the inlet } \\
\text { openings had no effect on the } \\
\text { flow rate. } \\
\text { - Strong gradients in tangential } \\
\text { velocity near the axis of the } \\
\text { nozzle due to the lack of the } \\
\text { central body. }\end{array}$ \\
\hline
\end{tabular}

\section{Conclusion}

- The application of supersonic separators in natural gas treatment has been studied and practiced for over two decades now. Several aspects of the technology including physical design, sensitivity to flow conditions, and various design features such as swirl generation and diffuser properties have been studied using analytical, numerical, and experimental techniques.

- Using the conventional method of separation, it is impossible to initiate condensation at pressures higher than 100atm. Besides, the condensation process might not be amenable to full control over a wider operating region. Therefore, it is necessary to rely on a separator that partly or entirely uses supersonic flow.

- Turndown flexibility is a critical requirement to cater for variation of mass flow rate. The reported designs fail to have this feature.

- Swirl generation device is also an important part of the design of these separators, and further research can potentially increase the knowledge base to improve the swirl generation effectiveness significantly.

- Many of the designs discussed above use straighteners in the substantially gaseous region. However, the decision on the geometry and location of this component is still not clear.

- The research on mechanical gas separators for the last few decades had studied the sizing of the supersonic zone, design of the swirler, design of the separation section, prediction of the gas property, condensation enhancement, and CFD simulation. 
- This paper reviewed the available supersonic separator designs and limitations in a very simple way, as well as making a brief comparison between them in terms of the main features and limitations.

\section{Abbreviation}

$\begin{array}{ll}\text { AGRS } & \text { Acid Gas Removal System } \\ { }^{\circ} \mathrm{C} & \text { Celsius } \\ \text { CFD } & \text { Computational Fluid Dynamics } \\ \text { LNG } & \text { Liquefied natural gas } \\ \text { SSB } & \text { Sarawak Shell Berhad }\end{array}$

\section{Symbols}

$\begin{array}{ll}D_{1} & \text { Inlet diameter } \\ D_{c r} & \text { Throat diameter } \\ D & \text { Convergent diameter } \\ D_{2} & \text { Outlet diameter } \\ L & \text { Length of the convergent nozzle } \\ L_{2} & \text { Length of the diverging nozzle } \\ X_{m} & \text { Convergent curve relative coordinate } \\ x & \text { Distance from the inlet to arbitrary cross section }\end{array}$

The authors would like to acknowledge Universiti Teknologi PETRONAS (UTP) financial assistance under Graduate Assistance (GA) Scheme.

\section{References}

1 M. S. Onn, A. A. Seman, Z. Kassim, and M. A. Esa, "CO ${ }_{2}$ Separation from Natural Gas Through Hydrate Formation," (2014).

2 R. A. Samawe, K. Rostani, A. Mohd Jalil, M. Esa, and N. Othman, "Concept Proofing of upersonic Nozzle Separator for $\mathrm{CO}_{2}$ Separation from Natural Gas using a Flow Loop," (2014).

3 S. Kong, J. Liu, L. Zhao, P. Cai, and Y. Huang, "Study of $\mathrm{CO}_{2}$ Separation Characteristic in Supersonic High-Pressure Vortex Tube," in Materials for Renewable Energy \& Environment (ICMREE), 2011 International Conference on, (2011), pp. 936-940.

4 T. González, M. Netušil, and P. Ditl, "Raw Gas Dehydration on Supersonic Swirling Separator," Czasopismo Tech. Mech. J., vol. 109, (2012).

5 C. Wen, X. Cao, J. Zhang, and L. Wu, "Three-dimensional Numerical Simulation of the Supersonic Swirling Separator," in The Twentieth International Offshore and Polar Engineering Conference, (2010).

6 C. Wen, X. Cao, Y. Yang, and J. Zhang, "Effects of Swirls on Natural Gas Flow in Supersonic Separators," in The Twenty-first International Offshore and Polar Engineering Conference, (2011).

7 F. T. Okimoto and J. M. Brouwer, "Supersonic Gas Conditioning," vol. 223 (2002).

8 H. C. Man, J. Duan, and T. M. Yue, "Design and Characteristic Analysis of Supersonic Nozzles for High Gas Pressure Laser Cutting," J. Mater. Process. Technol., vol. 63, pp. 217-222, (1997). 
9 C. Wen, X. Cao, and Y. Yang, "Swirling Flow of Natural Gas in Supersonic Separators," Chem. Eng. Process., vol. 50, pp. 644-649, (2011).

10 C. Wen, X. Cao, Y. Yang, and Y. Feng, "Prediction of Mass Flow Rate in Supersonic Natural Gas Processing," Oil Gas Sci. Technol.- Rev. IFP. J., vol. 70, pp. 1101-1109, (2013).

11 C. Wen, X. Cao, Y. Yang, and J. Zhang, "Supersonic Swirling Characteristics of Natural Gas in Convergent-Divergent Nozzles," Pet. Sci. J., vol. 8, pp. 114-119, (2011).

12 C. Wen, Y. Feng, X. Cao, Y. Yang, and P. Witt, "Effects of Operating Parameters on Flow Characteristics of Natural Gas in Supersonic Separators," in Offshore Technology Conference, (2013).

13 C. Wen, Y. Feng, P. Witt, X. Cao, and Y. Yang, "CFD Simulation of Supersonic Swirling Separation of Natural Gas Using a Delta Wing," in Proceedings of the Ninth International Conference on CFD in the Minerals and Process Industries, (2012).

14 Y. Yang, C. Wen, S. Wang, Y. Feng, and P. Witt, "The Swirling Flow Structure in Supersonic Separators for Natural Gas Dehydration," RSC ADV J., vol. 4, pp. 52967-52972, (2014).

K. Foelsch, "The Analytical Design of an Axially Symmetric Laval Nozzle for a Parallel and Uniform Jet," J. Aeronaut. Sci., (2012).

16 M. Betting, T. V. Holten, and J. M. H. M. V. Veen, "Supersonic Separator Apparatus and Method," US Patent US 20020194988A1, (2002).

17 B. Prast, R. A. van Dam, J. F. H. Willems, and M. E. H. van Dongen, "Formation of Nano-Sized Water Droplets in a Supersonic Expansion Flow," J. Aerosol Sci, vol. 27, pp. S147-S148, (1996).

18 P. Schinkelshoek and H. Epsom, "Supersonic Gas Conditioning-Low Pressure Drop Twister for NGL Recovery," (2006).

19 F. T. Okimoto, S. Sibani, and M. Lander, "Twister Supersonic Gas Conditioning Process," (2002).

20 B. Prast, P. Schinkelshoek, B. Lammers, and M. Betting, "CFD for Supersonic Gas Processing," in NEL Multiphase Separation and Multiphase Pumping Technologies Conference, (2005), pp. 53-58.

21 M. Haghighi, K. A. Hawboldt, and M. A. Abdi, "Supersonic Gas Separators: Review of Latest Developments," J NAT GAS SCI ENG, vol. 27, pp. 109-121, (2015).

22 V. Alfyorov, L. Bagirov, L. Dmitriev, V. Feygin, S. Imayev, and J. R. Lacey, "Supersonic Nozzle Efficiently Separates Natural Gas Components," Oil Gas J., vol. 103, pp. 53-58, (2005).

23 R. L. Garrett, "Supersonic Flow Seprator," US Patent 3559373, (1971).

24 S. Keisuk, "Separation of Gaseous Mixtures," Japan Patent JPH0217-921A, (1990).

25 V. Holten, "Method and Device for Separating a Gas from a Gas Mixture," (1992).

26 A. Borissov, G. Mirzoev, and V. Shtern, "Supersonic Swirling Separator 2 (Sustor2)," ed: Google Patents, (2014).

27 C. Wen, Y. Yang, and W. Jiang, "Supersonic Expansion Refrigeration and Cyclone Separation Device for Natural Gas," (2012).

28 L. Hengwei, L. Zhonggliang, F. Yongxun, G. Keyu, and Y. Tingmin, "Characteristic of a Supersonic Swirling Dehydration System of Natural Gas," Chin. J. Chem. Eng., vol. 13, pp. 9-12, (2005).

29 Z. Liu, J. Ding, W. Jiang, J. Zhang, and Y. Feng, "Numerical Simulation of HighlySwirling Supersonic Flow Inside a Laval Nozzle," PROG COMPUT FLUID DY, vol. 8, pp. 536-540, (2008). 